\title{
STOLARSKY'S INVARIANCE PRINCIPLE FOR PROJECTIVE SPACES
}

\author{
M.M. SKRIGANOV
}

\begin{abstract}
We show that Stolarsky's invariance principle, known for point distributions on the Euclidean spheres, can be extended to the real, complex, and quaternionic projective spaces and the octonionic projective plane. (The paper will be published in Journal of Complexity, 2020.)
\end{abstract}

\section{INTROUCTION AND MAIN RESULTS}

Let $S^{d}=\left\{x \in \mathbb{R}^{d+1}:\|x\|=1\right\}$ be the standard $d$-dimensional unit sphere in $\mathbb{R}^{d+1}$ with the geodesic (great circle) metric $\theta$ and the Lebesgue measure $\mu$ normalized by $\mu\left(S^{d}\right)=1$. We write $\mathcal{C}(y, t)=\left\{x \in S^{d}:(x, y)>t\right\}$ for the spherical cap of height $t \in[-1,1]$ centered at $y \in S^{d}$. Here we write $(\cdot, \cdot)$ and $\|\cdot\|$ for the inner product and the Euclidean norm in $\mathbb{R}^{d+1}$.

For an $N$-point subset $\mathcal{D}_{N} \subset S^{d}$, the spherical cap quadratic discrepancy is defined by

$$
\lambda^{c a p}\left[\mathcal{D}_{N}\right]=\int_{-1}^{1} \int_{S^{d}}\left(\#\left\{\mid \mathcal{C}(y, t) \cap \mathcal{D}_{N}\right\}-N \mu(\mathcal{C}(y, t))\right)^{2} \mathrm{~d} \mu(y) \mathrm{d} t .
$$

We introduce the sum of pairwise Euclidean distances between points of $\mathcal{D}_{N}$

$$
\tau\left[\mathcal{D}_{N}\right]=\frac{1}{2} \sum_{x_{1}, x_{2} \in \mathcal{D}_{N}}\left\|x_{1}-x_{2}\right\|=\sum_{x_{1}, x_{2} \in \mathcal{D}_{N}} \sin \frac{1}{2} \theta\left(x_{1}, x_{2}\right),
$$

and write $\langle\tau\rangle$ for the average value of the Euclidean distance on $S^{d}$,

$$
\langle\tau\rangle=\frac{1}{2} \iint_{S^{d} \times S^{d}}\left\|y_{1}-y_{2}\right\| d \mu\left(y_{1}\right) \mathrm{d} \mu\left(y_{2}\right) .
$$

The study of the quantities (1.1) and (1.2) falls within the subjects of discrepancy theory and geometry of distances, see [2,7] and references therein. It turns out that the quantities (1.1) and (1.2) are not independent and are intimately related by the following remarkable identity

$$
\gamma\left(S^{d}\right) \lambda^{c a p}\left[\mathcal{D}_{N}\right]+\tau\left[\mathcal{D}_{N}\right]=\langle\tau\rangle N^{2}
$$

for an arbitrary $N$-point subset $\mathcal{D}_{N} \subset S^{d}$. Here $\gamma\left(S^{d}\right)$ is a positive constant independent of $\mathcal{D}_{N}$,

$$
\gamma\left(S^{d}\right)=\frac{d \sqrt{\pi} \Gamma(d / 2)}{2 \Gamma((d+1) / 2)} \sim \sqrt{\pi d / 2}
$$

2010 Mathematics Subject Classification. 11K38, 22F30, 52C99.

Key words and phrases. Projective spaces, geometry of distances, discrepancies, spherical functions, Jacobi polynomials.

This work is supported by the Program of the Presidium of the Russian Academy of Sciences "New Methods of Mathematical Modeling in the Study of Nonlinear Dynamical Systems" under Grant PRAS 08-04. 
The identity (1.4) was established by Stolarsky [27, and known in the literature as Stolarsky's invariance principle. Its original proof has been essentially simplified by Brauchart and Dick [13. Further simplifications were given in the paper [10] by Bilyk, Dai and Matzke. Particularly, the explicit formula (1.5) has been given in 10, 13. In our notation $\gamma\left(S^{d}\right)=\left(2 C_{d}\right)^{-1}$, where $C_{d}$ is the constant in [10, Theorem 2.2] and [13, Eq. (6)].

In the present paper we consider the relations of this type in a more general setting. Let $\mathcal{M}$ be a compact metric measure space with a fixed metric $\theta$ and a finite Borel measure $\mu$, normalized, for convenience, by

$$
\operatorname{diam}(\mathcal{M}, \theta)=\pi, \quad \mu(\mathcal{M})=1
$$

where $\operatorname{diam}(\mathcal{E}, \rho)=\sup \left\{\rho\left(x_{1}, x_{2}\right): x_{1}, x_{2} \in \mathcal{E}\right\}$ denotes the diameter of a subset $\mathcal{E} \subseteq \mathcal{M}$ with respect to a metric $\rho$.

We write $\mathcal{B}(y, r)=\{x \in \mathcal{M}: \theta(x, y)<r\}$ for the ball of radius $r \in \mathcal{I}$ centered at $y \in \mathcal{M}$ and of volume $v(y, r)=\mu(\mathcal{B}(y, r))$. Here $\mathcal{I}=\left\{r=\theta\left(x_{1}, x_{2}\right): x_{1}, x_{2} \in \mathcal{M}\right\}$ denotes the set of all possible radii. If the space $\mathcal{M}$ is connected, we have $\mathcal{I}=[0, \pi]$.

We consider distance-invariant metric spaces. Recall that a metric space $\mathcal{M}$ is called distance-invariant, if the volume of any ball $v(r)=v(y, r)$ is independent of $y \in \mathcal{M}$, see [23. p. 504]. The typical examples of distance-invariant spaces are homogeneous spaces $\mathcal{M}=G / K$, where $G$ is a compact group, $K \subset G$ is a closed subgroup, and a metric $\theta$ and a measure $\mu$ on $\mathcal{M}$ are $G$-invariant.

For an $N$-point subset $\mathcal{D}_{N} \subset \mathcal{M}$, the ball quadratic discrepancy is defined by

$$
\left.\lambda\left[\xi, \mathcal{D}_{N}\right]=\int_{\mathcal{I}} \int_{\mathcal{M}}\left(\#\left\{\mathcal{B}(y, r) \cap \mathcal{D}_{N}\right\}-N v(r)\right)\right)^{2} \mathrm{~d} \mu(y) \mathrm{d} \xi(r),
$$

where $\xi$ is a measure on the set of radii $\mathcal{I}$.

Notice that for $S^{d}$ spherical caps and balls are related by $\mathcal{C}(y, t)=\mathcal{B}(y, r)$, $t=\cos r$, and the discrepancies (1.1) and (1.7) are related by $\lambda^{\operatorname{cap}}\left[\mathcal{D}_{N}\right]=\lambda\left[\xi^{\natural}, \mathcal{D}_{N}\right]$, where $\mathrm{d} \xi^{\natural}(r)=\sin r \mathrm{~d} r, r \in \mathcal{I}=[0, \pi]$.

The ball quadratic discrepancy (1.7) can be written in the form

$$
\lambda\left[\xi, \mathcal{D}_{N}\right]=\sum_{x_{1}, x_{2} \in \mathcal{D}_{N}} \lambda\left(\xi, x_{1}, x_{2}\right)
$$

with the kernel

$$
\lambda\left(\xi, x_{1}, x_{2}\right)=\int_{\mathcal{I}} \int_{\mathcal{M}} \Lambda\left(\mathcal{B}(y, r), x_{1}\right) \Lambda\left(\mathcal{B}(y, r), x_{2}\right) \mathrm{d} \mu(y) \mathrm{d} \xi(r),
$$

where

$$
\Lambda(\mathcal{B}(y, r), x)=\chi(\mathcal{B}(y, r), x)-v(r),
$$

and $\chi(\mathcal{E}, \cdot)$ denotes the characteristic function of a subset $\mathcal{E} \subseteq \mathcal{M}$.

The symmetry of the metric $\theta$ implies the following relation

$$
\chi(\mathcal{B}(y, r), x)=\chi(\mathcal{B}(x, r), y)=\chi_{0}(r-\theta(x, y)),
$$

where $\chi_{0}(\cdot)$ is the characteristic function of the half-axis $(0, \infty)$. Substituting (1.10) into (1.9) and using (1.11), we obtain

$$
\lambda\left(\xi, x_{1}, x_{2}\right)=\int_{\mathcal{I}}\left(\mu\left(\mathcal{B}\left(x_{1}, r\right) \cap \mathcal{B}\left(x_{2}, r\right)\right)-v(r)^{2}\right) \mathrm{d} \xi(r)
$$

For an arbitrary metric $\rho$ on $\mathcal{M}$ we introduce the sum of pairwise distances

$$
\rho\left[\mathcal{D}_{N}\right]=\sum_{x_{1}, x_{2} \in \mathcal{D}_{N}} \rho\left(x_{1}, x_{2}\right)
$$


and the average value

$$
\langle\rho\rangle=\int_{\mathcal{M} \times \mathcal{M}} \rho\left(y_{1}, y_{2}\right) \mathrm{d} \mu\left(y_{1}\right) \mathrm{d} \mu\left(y_{2}\right) .
$$

We introduce the following symmetric difference metrics on the space $\mathcal{M}$

$$
\begin{aligned}
\theta^{\Delta}\left(\xi, y_{1}, y_{2}\right) & =\frac{1}{2} \int_{\mathcal{I}} \mu\left(\mathcal{B}\left(y_{1}, r\right) \Delta \mathcal{B}\left(y_{2}, r\right)\right) \mathrm{d} \xi(r) \\
& =\frac{1}{2} \int_{\mathcal{I}} \int_{\mathcal{M}} \chi\left(\mathcal{B}\left(y_{1}, r\right) \Delta \mathcal{B}\left(y_{2}, r\right), y\right) \mathrm{d} \mu(y) \mathrm{d} \xi(r)
\end{aligned}
$$

where

$$
\mathcal{B}\left(y_{1}, r\right) \Delta \mathcal{B}\left(y_{2}, r\right)=\mathcal{B}\left(y_{1}, r\right) \cup \mathcal{B}\left(y_{2}, r\right) \backslash \mathcal{B}\left(y_{1}, r\right) \cap \mathcal{B}\left(y_{2}, r\right)
$$

is the symmetric difference of the balls $\mathcal{B}\left(y_{1}, r\right)$ and $\mathcal{B}\left(y_{2}, r\right)$. We have

$$
\chi\left(\mathcal{B}\left(y_{1}, r\right) \Delta \mathcal{B}\left(y_{2}, r\right), y\right)=\left|\chi\left(\mathcal{B}\left(y_{1}, r\right), y\right)-\chi\left(\mathcal{B}\left(y_{2}, r\right), y\right)\right| .
$$

Therefore,

$$
\theta^{\Delta}\left(\xi, y_{1}, y_{2}\right)=\frac{1}{2} \int_{\mathcal{I}} \int_{\mathcal{M}}\left|\chi\left(\mathcal{B}\left(y_{1}, r\right), y\right)-\chi\left(\mathcal{B}\left(y_{2}, r\right), y\right)\right| \mathrm{d} \mu(y) \mathrm{d} \xi(r) .
$$

On the other hand, we have

$$
\begin{aligned}
& \chi\left(\mathcal{B}\left(y_{1}, r\right) \Delta \mathcal{B}\left(y_{2}, r\right)\right) \\
& =\chi\left(\mathcal{B}\left(y_{1}, r\right), y\right)+\chi\left(\mathcal{B}\left(y_{2}, r\right), y\right)-2 \chi\left(\mathcal{B}\left(y_{1}, r\right), y\right) \chi\left(\mathcal{B}\left(y_{2}, r\right), y\right) .
\end{aligned}
$$

Substituting (1.18) into (1.15) and using (1.11), we obtain

$$
\theta^{\Delta}\left(\xi, y_{1}, y_{2}\right)=\int_{\mathcal{I}}\left(v(r)-\mu\left(\mathcal{B}\left(y_{1}, r\right) \cap \mathcal{B}\left(y_{2}, r\right)\right)\right) \mathrm{d} \xi(r),
$$

and

$$
\left\langle\theta^{\Delta}(\xi)\right\rangle=\int_{\mathcal{I}}\left(v(r)-v(r)^{2}\right) \mathrm{d} \xi(r) .
$$

In line with the definition (1.13), we put

$$
\theta^{\Delta}\left[\xi, \mathcal{D}_{N}\right]=\sum_{x_{1}, x_{2} \in \mathcal{D}_{N}} \theta^{\Delta}\left(\xi, x_{1}, x_{2}\right) .
$$

Comparing the relations (1.12), (1.19), and (1.20), we arrive at the following.

Proposition 1.1. Let a compact metric measure space $\mathcal{M}$ with a metric $\theta$ and a measure $\mu$ be distance-invariant. Then we have

$$
\lambda\left(\xi, y_{1}, y_{2}\right)+\theta^{\Delta}\left(\xi, y_{1}, y_{2}\right)=\left\langle\theta^{\Delta}(\xi)\right\rangle .
$$

In particular, we have the following $L_{1}$-invariance principle

$$
\lambda\left[\xi, \mathcal{D}_{N}\right]+\theta^{\Delta}\left[\xi, \mathcal{D}_{N}\right]=\left\langle\theta^{\Delta}(\xi)\right\rangle N^{2}
$$

for an arbitrary $N$-point subset $\mathcal{D}_{N} \subset \mathcal{M}$.

The identities (1.21) and (1.22) hold with any measure $\xi$ on the set of radii $\mathcal{I}$ such that the integrals (1.12), (1.19) and (1.20) converge (for example, with any finite measure $\xi$ ). 
Other versions and applications of this result can be found in 24].

Recall that a metric space $\mathcal{M}$ with a metric $\rho$ is called isometrically $L_{q}$-embeddable $(q=1$ or 2$)$, if there exists a map $\varphi: \mathcal{M} \ni x \rightarrow \varphi(x) \in L_{q}$, such that $\rho\left(x_{1}, x_{2}\right)=$ $\left\|\varphi\left(x_{1}\right)-\varphi\left(x_{2}\right)\right\|_{L_{q}}$ for all $x_{1}, x_{2} \in \mathcal{M}$. Notice that the $L_{2}$-embeddability is stronger and implies the $L_{1}$-embeddability, see [17, Sec. 6.3].

Since the space $\mathcal{M}$ is isometrically $L_{1}$-embeddable with respect to the symmetric difference metrics $\theta^{\Delta}(\xi)$, see (1.17), the identity (1.22) is called the $L_{1}$-invariance principle. At the same time, Stolarsky's invariance principle should be called the $L_{2}$-invariance principle, because it involves the Euclidean metric.

In the present paper we shall prove the $L_{2}$-invariance principles for compact Riemannian symmetric manifolds of rank one. All these manifolds are completely classified, see, for example, [8, Chap.3] and [30, Sec. 8.12]. They are homogeneous spaces $\mathcal{M}=G / K$, where $G$ and $K \subset G$ are compact Lie groups. The complete list of all compact Riemannian symmetric manifolds of rank one is the following:

(i) The $d$-dimensional Euclidean spheres $S^{d}=S O(d+1) / S O(d) \times\{1\}, d \geqslant 2$, and $S^{1}=O(2) / O(1) \times\{1\}$.

(ii) The real projective spaces $\mathbb{R} P^{n}=O(n+1) / O(n) \times O(1)$.

(iii) The complex projective spaces $\mathbb{C} P^{n}=U(n+1) / U(n) \times U(1)$.

(iv) The quaternionic projective spaces $\mathbb{H} P^{n}=S p(n+1) / S p(n) \times S p(1)$,

(v) The octonionic projective plane $\mathbb{O} P^{2}=F_{4} / \operatorname{Spin}(9)$.

Here we use the standard notation from the theory of Lie groups; in particular, $F_{4}$ is one of the exceptional Lie groups in Cartan's classification.

The indicated projective spaces $\mathbb{F} P^{n}$ as compact Riemannian manifolds have dimensions $d$,

$$
d=\operatorname{dim}_{\mathbb{R}} \mathbb{F} P^{n}=n d_{0}, \quad d_{0}=\operatorname{dim}_{\mathbb{R}} \mathbb{F},
$$

where $d_{0}=1,2,4,8$ for $\mathbb{F}=\mathbb{R}, \mathbb{C}, \mathbb{H}, \mathbb{O}$, correspondingly.

For the spheres $S^{d}$ we put $d_{0}=d$ by definition. Projective spaces of dimension $d_{0}(n=1)$ are homeomorphic to the spheres $S^{d_{0}}: \mathbb{R} P^{1} \approx S^{1}, \mathbb{C} P^{1} \approx S^{2}, \mathbb{H} P^{1} \approx$ $S^{4}, \mathbb{O} P^{1} \approx S^{8}$. We can conveniently agree that $d>d_{0}(n \geqslant 2)$ for projective spaces, while the equality $d=d_{0}$ holds only for spheres. Under this convention, the dimensions $d=n d_{0}$ and $d_{0}$ define uniquely (up to homeomorphisms) the corresponding homogeneous space which we denote by $Q=Q\left(d, d_{0}\right)$.

We consider $Q\left(d, d_{0}\right)$ as a metric measure space with the metric $\theta$ and measure $\mu$ proportional to the invariant Riemannian distance and measure on $Q\left(d, d_{0}\right)$. The coefficients of proportionality are defined to satisfy (1.6). In what follows we always assume that $n=2$ if $\mathbb{F}=\mathbb{O}$, since projective spaces $\mathbb{O} P^{n}$ do not exist for $n>2$.

The spaces $Q\left(d, d_{0}\right)$ have a very rich geometrical structure and can be also characterized as compact connected two-point homogeneous spaces. This means that for any two pairs of points $x_{1}, x_{2}$ and $y_{1}, y_{2}$ in $Q\left(d, d_{0}\right)$ with $\theta\left(x_{1}, x_{2}\right)=\theta\left(y_{1}, y_{2}\right)$ there exists an isometry $g \in G$, such that $y_{1}=g x_{1}, y_{2}=g x_{2}$. In more detail the geometry of spaces $\mathbb{F} P^{n}$ will be outlined in Section 2 .

Any space $Q\left(d, d_{0}\right)$ is distance-invariant and the volume of balls is given by

$$
v(r)=B\left(d / 2, d_{0} / 2\right)^{-1} \int_{0}^{r}\left(\sin \frac{1}{2} u\right)^{d-1}\left(\cos \frac{1}{2} u\right)^{d_{0}-1} \mathrm{~d} u, \quad r \in[0, \pi],
$$

where $B(\cdot, \cdot)$ is the beta function, see (4.10). Equivalent forms of (1.24) can be found in the literature, see [18, pp. 177-178], [22, pp. 165-168], 23, pp. 508-510]. 
The chordal metric on the spaces $Q\left(d, d_{0}\right)$ can be defined by

$$
\tau\left(x_{1}, x_{2}\right)=\sin \frac{1}{2} \theta\left(x_{1}, x_{2}\right), \quad x_{1}, x_{2} \in Q\left(d, d_{0}\right) .
$$

Notice that the expression (1.25) defines a metric because the function $\varphi(\theta)=$ $\sin \theta / 2,0 \leqslant \theta \leqslant \pi$, is concave, increasing, and $\varphi(0)=0$, that implies the triangle inequality. For the sphere $S^{d}$ we have $\cos \theta\left(x_{1}, x_{2}\right)=\left(x_{1}, x_{2}\right), x_{1}, x_{2} \in S^{d}$ and

$$
\tau\left(x_{1}, x_{2}\right)=\sin \frac{1}{2} \theta\left(x_{1}, x_{2}\right)=\frac{1}{2}\left\|x_{1}-x_{2}\right\| .
$$

Each projective space $\mathbb{F} P^{n}$ can be canonically embedded into the unit sphere

$$
\Pi: Q\left(d, d_{0}\right) \ni x \rightarrow \Pi(x) \in S^{m-1} \subset \mathbb{R}^{m}, \quad m=\frac{1}{2}(n+1)(d+2),
$$

such that

$$
\tau\left(x_{1}, x_{2}\right)=\frac{1}{\sqrt{2}}\left\|\Pi\left(x_{1}\right)-\Pi\left(x_{2}\right)\right\|, \quad x_{1}, x_{2} \in \mathbb{F} P^{n},
$$

where $\|\cdot\|$ is the Euclidean norm in $\mathbb{R}^{m}$. Hence, the metric $\tau\left(x_{1}, x_{2}\right)$ is proportional to the Euclidean length of a segment joining the corresponding points $\Pi\left(x_{1}\right)$ and $\Pi\left(x_{2}\right)$ on the unit sphere and normalized by $\operatorname{diam}\left(Q\left(d, d_{0}\right), \tau\right)=1$. The embedding (1.27) will be described explicitly in Section 2,

The chordal metric $\tau$ on the complex projective space $\mathbb{C} P^{n}$ is known as the Fubini-Study metric. The chordal metric on projective spaces has been discussed in the papers [14,15] in connection with special point configurations in such spaces. The chordal metric has been also defined for Grassmannian manifolds in [16].

Now we are in position to state our main result.

Theorem 1.1. For any space $Q=Q\left(d, d_{0}\right)$ the chordal metric (1.25) and the symmetric difference metric (1.15) are related by

$$
\tau\left(x_{1}, x_{2}\right)=\gamma(Q) \theta^{\Delta}\left(\xi^{\natural}, x_{1}, x_{2}\right), x_{1}, x_{2} \in Q,
$$

where $\mathrm{d} \xi^{\natural}(r)=\sin r \mathrm{~d} r, r \in[0, \pi]$, and

$$
\gamma(Q)=\frac{\langle\tau\rangle}{\left\langle\theta^{\Delta}\left(\xi^{\natural}\right)\right\rangle}=\frac{\operatorname{diam}(Q, \tau)}{\operatorname{diam}\left(Q, \theta^{\Delta}\left(\xi^{\natural}\right)\right)} .
$$

The proof of Theorem 1.1 is given in Section 3. It is clear that the equalities (1.30) follow immediately from (1.29). It suffices to calculate the average values (1.14) of both metrics in (1.29) to obtain the first equality in (1.30). Similarly, writing (1.29) for any pair of antipodal points $x_{1}, x_{2}, \theta\left(x_{1}, x_{2}\right)=\pi$, we obtain the second equality in (1.30). Recall that points $x_{1}, x_{2}$ are antipodal for a metric $\rho$ if $\rho\left(x_{1}, x_{2}\right)=\operatorname{diam}(Q, \rho)$. If points $x_{1}, x_{2}$ are antipodal for the metric $\theta$, then, in view of (1.25) and (1.29), they are also antipodal for the metrics $\tau$ and $\theta^{\Delta}\left(\eta^{\natural}\right)$.

Comparing Theorem 1.1 and Proposition 1.1, we arrive at the following.

Corollary 1.1. For any space $Q=Q\left(d, d_{0}\right)$ we have the $L_{2}$-invariance principle

$$
\gamma(Q) \lambda\left[\xi^{\natural}, \mathcal{D}_{N}\right]+\tau\left[\mathcal{D}_{N}\right]=\langle\tau\rangle N^{2}
$$

for an arbitrary $N$-point subset $\mathcal{D}_{N} \subset Q$.

The identity (1.31) can be thought of as an extension of Stolarsky's invariance principle to all compact Riemannian symmetric manifolds of rank one. 
Now we wish to calculate the constants $\langle\tau\rangle$ and $\gamma(Q)$ in the invariance principle (1.31). Using (1.24), (1.25), and the formula (4.10) for the beta function, we immediately obtain

$$
\langle\tau\rangle=B\left(d / 2, d_{0} / 2\right)^{-1} B\left((d+1) / 2, d_{0} / 2\right) .
$$

The explicit calculation of the constant $\gamma\left(\mathbb{F} P^{n}\right)$ is more differentiated. In principle, for this purpose, one can use the first equality in (1.30) and the formula (1.20). In the case of $\mathbb{F} P^{n}, \mathbb{F} \neq \mathbb{R}$, the integrals (1.24) have rather simple explicit expressions, see [19, p. 341]. To calculate the constant $\gamma\left(\mathbb{R} P^{n}\right)$ one can use the integral representation (3.10) given below. However, such calculations turn out to be rather cumbersome. In the present paper, we shall use a more general approach relying on the theory of spherical functions on the spaces $Q\left(d, d_{0}\right)$ to obtain the following.

Theorem 1.2. For any space $Q\left(d, d_{0}\right)$, we have

$$
\gamma\left(Q\left(d, d_{0}\right)\right)=\frac{\sqrt{\pi}}{4}\left(d+d_{0}\right) \frac{\Gamma\left(d_{0} / 2\right)}{\Gamma\left(\left(d_{0}+1\right) / 2\right)}=\frac{d+d_{0}}{2 d_{0}} \gamma\left(S^{d_{0}}\right),
$$

where $\gamma\left(S^{d_{0}}\right)$ is defined by (1.5).

Theorem 1.2 is proved in section 4 . For the sphere $S^{d}=Q(d, d)$, the relation (1.32) coincides with the formula (1.5). For projective spaces, from (1.32) we obtain the following.

Corollary 1.2. For projective spaces $\mathbb{F} P^{n}=Q\left(n d_{0}, d_{0}\right), d_{0}=\operatorname{dim}_{\mathbb{R}} \mathbb{F}$, we have

$$
\gamma\left(\mathbb{F} P^{n}\right)=\frac{n+1}{2} \gamma\left(S^{d_{0}}\right)
$$

and therefore,

$$
\left.\begin{array}{l}
\gamma\left(\mathbb{R} P^{n}\right)=\frac{n+1}{2} \gamma\left(S^{1}\right)=\frac{\pi}{4}(n+1), \\
\gamma\left(\mathbb{C} P^{n}\right)=\frac{n+1}{2} \gamma\left(S^{2}\right)=n+1, \\
\gamma\left(\mathbb{H} P^{n}\right)=\frac{n+1}{2} \gamma\left(S^{4}\right)=\frac{4}{3}(n+1), \\
\gamma\left(\mathbb{O} P^{2}\right)=\frac{3}{2} \gamma\left(S^{8}\right)=\frac{192}{35} .
\end{array}\right\}
$$

Notice that the comparison of the formulas (1.5) with (1.33) and (1.34) shows that for spheres and projective spaces the behavior of the constants $\gamma\left(Q\left(d, d_{0}\right)\right)$ differs essentially in large dimensions.

In conclusion of this section, we briefly describe applications of the invariance principle (1.31) to uniform point distributions in the spaces $Q=Q\left(d, d_{0}\right)$. With the help of the invariance principle (1.36), the results of distance geometry can be transformed to the corresponding results of discrepancy theory and vice versa.

Let us consider the following extremal quadratic discrepancies and sums of pairwise chordal distances

$$
\lambda_{N}(Q)=\inf _{\mathcal{D}_{N}} \lambda\left[\xi^{\natural}, \mathcal{D}_{N}\right], \quad \tau_{N}(Q)=\sup _{\mathcal{D}_{N}} \tau\left[\mathcal{D}_{N}\right],
$$

where the infimum and supremum are taken over all $N$-point subsets $\mathcal{D}_{N} \subset Q$. These quantities can be thought of as geometrical characteristics of the spaces $Q$.

From (1.31), we obtain the identity

$$
\gamma(Q) \lambda_{N}(Q)+\tau_{N}(Q)=\langle\tau\rangle N^{2} .
$$


First of all, the identity (1.36) implies the non-trivial inequality $\tau_{N}(Q) \leqslant\langle\tau\rangle N^{2}$. This inequality can be essentially improved. We have the upper bound

$$
\langle\tau\rangle N^{2}-\tau_{N}(Q) \lesssim N^{1-\frac{1}{d}},
$$

and by (1.36), we obtain

$$
\lambda_{N}(Q) \lesssim N^{1-\frac{1}{d}}
$$

On the other hand, we have the lower bound

$$
\lambda_{N}(Q) \gtrsim N^{1-\frac{1}{d}}
$$

and by (1.36), we obtain

$$
\langle\tau\rangle N^{2}-\tau\left[\mathcal{D}_{N}\right] \gtrsim N^{1-\frac{1}{d}}
$$

Combining the above bounds (1.37) - 1.40), we find the sharp orders of the extremal quantities (1.35)

$$
\left.\begin{array}{l}
\langle\tau\rangle N^{2}-\tau_{N}(Q) \simeq N^{1-\frac{1}{d}}, \\
\lambda_{N}(Q) \simeq N^{1-\frac{1}{d}} .
\end{array}\right\}
$$

For the spheres $S^{d}$, the upper bounds (1.37) and (1.38) have been established by Alexander [1] and Stolarsky [27, while the lower bounds (1.39) and (1.40) have been established by Beck [6], see also [2,7]. For all spaces $Q\left(d, d_{0}\right)$ the bounds (1.37) - (1.38) were proved in [25. Notice that the upper bounds of the type (1.37) and (1.38) can be established for very general compact metric measure spaces, see [12, 24, 26]. At the same time, the lower bounds (1.39) and (1.40) are much more specific. Their proof relies on harmonic analysis on the homogeneous spaces $Q\left(d, d_{0}\right)$, see [25], and can not be extended to general compact metric measure spaces.

The paper is organized as follows. In Section 2 we define and discuss the chordal metrics on the projective spaces $\mathbb{F} P^{n}, \mathbb{F}=\mathbb{R}, \mathbb{C}, \mathbb{H}, n \geqslant 2$, and the octonionic projective plane $\mathbb{O} P^{2}$ in terms of special models for such spaces. For the reader's convenience, we describe these models in close detail and give the necessary references. In Section 3 we prove Theorem 1.1 relying on the results of Section 2 and a special representation for symmetric difference metrics (Lemma 3.1). For completeness, in Section 3 we give a short proof of Stolarsky's invariance principle for the spheres $S^{d}$. A very short proof of Theorem 1.1 can be given in the special case of real projective spaces. This simple proof, specific for $\mathbb{R} P^{n}$, is also given in Section 3. In Section 4 we calculate the constants $\gamma\left(Q\left(d, d_{0}\right)\right)$ and prove Theorem 1.2. The proof relies on the zonal spherical function expansions for the chordal and symmetric difference metrics (Lemmas 4.1 and 4.2). In conclusion of Section 4, we briefly discuss explicit formulas for integrals with Jacobi polynomials which follow from our calculations (Remark 4.2).

\section{MOdELS OF PROJECTIVE SPACES AND CHORDAL METRICS}

Recall the general facts on the division algebras $\mathbb{R}, \mathbb{C}, \mathbb{H}, \mathbb{O}$ over the field of real numbers. We have the natural inclusions $\mathbb{R} \subset \mathbb{C} \subset \mathbb{H} \subset \mathbb{O}$, where the octonions (1) are a nonassociative and noncommutative algebra of dimension 8 with a basis $\left\{1, e_{1}, e_{2}, e_{3}, e_{4}, e_{5}, e_{6}, e_{7}\right\}$ (the multiplication table for these elements can be found, for example, in [5, p. 150] and [8, p. 90]), the quaternions $\mathbb{H}$ are an associative but noncommutative subalgebra of dimension 4 spanned by $\left\{1, e_{1}, e_{2}, e_{3}\right\}$, finally, $\mathbb{C}$ and 
$\mathbb{R}$ are associative and commutative subalgebras of dimensions 2 and 1 spanned by $\left\{1, e_{1}\right\}$ and $\{1\}$. From the multiplication table one can easily see that for any two indexes $1 \leqslant i, j \leqslant 7, i \neq j$, there exists an index $1 \leqslant k \leqslant 7$, such that

$$
e_{i} e_{j}=-e_{j} e_{i}=e_{k}, \quad i<j, \quad e_{i}^{2}=-1 .
$$

Let $a=\alpha_{0}+\sum_{i=1}^{7} \alpha_{i} e_{i}, \alpha_{i} \in \mathbb{R}, 0 \leqslant i \leqslant 7$, be a typical octonion. We write $\operatorname{Re} a=$ $\alpha_{0}$ for the real part, $\bar{a}=\alpha_{0}-\sum_{i=1}^{7} \alpha_{i} e_{i}$ for the conjugation, $|a|=\left(\alpha_{0}^{2}+\sum_{i-1}^{7} \alpha_{i}^{2}\right)^{1 / 2}$ for the norm. Using (2.1), one can easily check that

$$
\operatorname{Re} a b=\operatorname{Re} b a, \quad \overline{a b}=\overline{b a}, \quad|a|^{2}=a \bar{a}=\bar{a} a, \quad|a b|=|a||b| .
$$

Notice that by a theorem of Artin a subalgebra in $\mathbb{O}$ generated by any two octonions is associative and isomorphic to one of the algebras $\mathbb{H}, \mathbb{C}$, or $\mathbb{R}$, see $[5]$.

The usual model of projective spaces over the associative algebras $\mathbb{F}=\mathbb{R}, \mathbb{C}, \mathbb{H}$ is the following, see $5,8,20,30$. Let $\mathbb{F}^{n+1}$ be a linear space of vectors $\mathbf{a}=\left(a_{0}, \ldots, a_{n}\right)$, $a_{i} \in \mathbb{F}, 0 \leqslant i \leqslant n$ with the right multiplication by scalars $a \in \mathbb{F}$, the Hermitian inner product

and the norm $|\mathbf{a}|$,

$$
(\mathbf{a}, \mathbf{b})=\sum_{i=0}^{n} \bar{a}_{i} b_{i}, \quad \mathbf{a}, \mathbf{b} \in \mathbb{F}^{n+1},
$$

$$
|\mathbf{a}|^{2}=(\mathbf{a}, \mathbf{a})=\sum_{i=0}^{n}\left|a_{i}\right|^{2} .
$$

In view of associativity of the algebras $\mathbb{F}=\mathbb{R}, \mathbb{C}, \mathbb{H}$, a projective space $\mathbb{F} P^{n}$ can be defined as a set of one-dimensional (over $\mathbb{F}$ ) subspaces in $\mathbb{F}^{n+1}$ :

$$
\mathbb{F} P^{n}=\left\{p(\mathbf{a})=\mathbf{a} \mathbb{F}: \mathbf{a} \in \mathbb{F}^{n+1},|\mathbf{a}|=1\right\} .
$$

The metric $\theta$ on $\mathbb{F} P^{n}$ is defined by

$$
\cos \frac{1}{2} \theta(\mathbf{a}, \mathbf{b})=|(\mathbf{a}, \mathbf{b})|, \quad \mathbf{a}, \mathbf{b} \in \mathbb{F}^{n+1}, \quad|\mathbf{a}|=|\mathbf{b}|=1,0 \leqslant \theta(\mathbf{a}, \mathbf{b}) \leqslant \pi .
$$

In other words, $\frac{1}{2} \theta(\mathbf{a}, \mathbf{b})$ is the angle between the subspaces $p(\mathbf{a})$ and $p(\mathbf{b})$. The transitive group of isometries $U(n+1, \mathbb{F})$ for the metric $\theta$ consists of nondegenerate linear transformations of the space $\mathbb{F}^{n+1}$, preserving the inner product (2.3), and the stabilizer of a point is isomorphic to the subgroup $U(n, \mathbb{F}) \times U(1, \mathbb{F})$. Hence,

$$
\mathbb{F} P^{n}=U(n+1, \mathbb{F}) / U(n, \mathbb{F}) \times U(1, \mathbb{F}) .
$$

The groups $U(n+1, \mathbb{F})$ are indicated in Section 1 .

There is another model where a projective space $\mathbb{F} P^{n}, \mathbb{F}=\mathbb{R}, \mathbb{C}, \mathbb{H}$, is identified with the set of orthogonal projectors onto the subspaces $p(\mathbf{a}) \subset \mathbb{F}^{n+1}$. This model admits a generalization to the octonionic projective plane $\mathbb{O} P^{2}$ and in its terms the chordal metric can be naturally defined for all such projective spaces.

Let $\mathcal{H}\left(\mathbb{F}^{n+1}\right)$ denote the set of all Hermitian $(n+1) \times(n+1)$ matrices with the entries in $\mathbb{F}, \mathbb{F}=\mathbb{R}, \mathbb{C}, \mathbb{H}, \mathbb{O}$,

$$
\mathcal{H}\left(\mathbb{F}^{n+1}\right)=\left\{A=\left(\left(a_{i j}\right)\right): a_{i j}=\bar{a}_{j i}, a_{i j} \in \mathbb{F}, 0 \leqslant i, j \leqslant n\right\},
$$

where $n=2$ if $\mathbb{F}=\mathbb{O}$. It is clear that $\mathcal{H}\left(\mathbb{F}^{n+1}\right)$ is a linear space over $\mathbb{R}$ of dimension

$$
m=\operatorname{dim}_{\mathbb{R}} \mathcal{H}\left(\mathbb{F}^{n+1}\right)=\frac{1}{2}(n+1)(d+2), \quad d=n d_{0} .
$$

The space $\mathcal{H}\left(\mathbb{F}^{n+1}\right)$ is equipped with the symmetric real-valued inner product

$$
\langle A, B\rangle=\frac{1}{2} \operatorname{Tr}(A B+B A)=\operatorname{Re} \operatorname{Tr} A B=\operatorname{Re} \sum_{i, j=0}^{n} a_{i j} \overline{b_{i j}}
$$


and the Hilbert - Schmidt norm

$$
\|A\|=\left(\operatorname{Tr} A^{2}\right)^{1 / 2}=\left(\sum_{i, j=0}^{n}\left|a_{i j}\right|^{2}\right)^{1 / 2},
$$

where $\operatorname{Tr} A=\sum_{i=0}^{n} a_{i i}$ denotes the trace of a matrix $A$. For the distance $\|A-B\|$ between two matrices $A, B \in \mathcal{H}\left(\mathbb{F}^{n+1}\right)$, we have

$$
\|A-B\|^{2}=\|A\|^{2}+\|B\|^{2}-2\langle A, B\rangle .
$$

Thus, $\mathcal{H}\left(\mathbb{F}^{n+1}\right)$ can be thought of as the $m$-dimensional Euclidean space.

If $\mathbb{F} \neq \mathbb{O}$, the orthogonal projector $\Pi_{\mathbf{a}} \in \mathcal{H}\left(\mathbb{F}^{n+1}\right)$ onto $p(\mathbf{a})=\mathbf{a} \mathbb{F}$, $\mathbf{a}=$ $\left(a_{0}, \ldots, a_{n}\right) \in \mathbb{F}^{n+1},|\mathbf{a}|=1$, can be given by $\Pi_{\mathbf{a}}=\mathbf{a}(\mathbf{a}, \cdot)$ or as the $(n+1) \times(n+1)$ matrix with entries $\left(\Pi_{\mathbf{a}}\right)_{i, j}=a_{i} \bar{a}_{j}, 0 \leqslant i, j \leqslant n$. Therefore, the projective space (2.5) can be written as follows

$$
\mathbb{F} P^{n}=\left\{\Pi \in \mathcal{H}\left(\mathbb{F}^{n+1}\right): \Pi^{2}=\Pi, \operatorname{Tr} \Pi=1\right\} .
$$

One can easily check that for such projectors the inner products (2.3) and 2.10) are related by $\left\langle\Pi_{\mathbf{a}}, \Pi_{\mathbf{b}}\right\rangle=|(\mathbf{a}, \mathbf{b})|^{2}$, see [15, Eq. (2.1)], and the group of isometries $U(n+1, \mathbb{F})$ acts on such projectors by the formula $g(\Pi)=g \Pi g^{-1}, g \in U(n+1, \mathbb{F})$.

For the octonionic projective plane $\mathbb{O} P^{2}$ a similar model is also true. A detailed discussion of this model can be found in [5, 8, 20], including an explanation why octonionic projective spaces $\mathbb{O} P^{n}$ do not exist if $n>2$. In this model one puts by definition

$$
\mathbb{O} P^{2}=\left\{\Pi \in \mathcal{H}\left(\mathbb{O}^{3}\right): \Pi^{2}=\Pi, \operatorname{Tr} \Pi=1\right\} .
$$

The formulas (2.13) and (2.14) are quite similar. One can check that each matrix in (2.14) can be written as $\Pi_{\mathbf{a}} \in \mathbb{O} P^{2}$ for a triple $\mathbf{a}=\left(a_{0}, a_{1}, a_{2}\right) \in \mathbb{O}^{3}$, where $\left(\Pi_{\mathbf{a}}\right)_{i, j}=a_{i} \bar{a}_{j}, 0 \leqslant i, j \leqslant 2,|\mathbf{a}|^{2}=\left|a_{0}\right|^{2}+\left|a_{1}\right|^{2}+\left|a_{2}\right|^{2}=1$, and additionally $\left(a_{0} a_{1}\right) a_{2}=a_{0}\left(a_{1} a_{2}\right)$, see [20, Lemma 14.90]. The additional condition means that the subalgebra in $\mathbb{O}$ generated by the elements $a_{0}, a_{1}, a_{2}$ is associative. Using this fact, one can show that $\mathbb{O} P^{2}$ is a 16 -dimensional compact connected Riemannian manifold, see [5, 8, 20].

The group of nondegenerate linear transformations $g$ of the space $\mathcal{H}\left(\mathbb{O}^{3}\right)$ preserving the squares $g\left(A^{2}\right)=g(A)^{2}, A \in \mathcal{H}\left(\mathbb{O}^{3}\right)$, is isomorphic to the 52-dimensional exceptional Lie group $F_{4}$. This group also preserves the trace, inner product (2.10) and norm (2.11) of matrices $A \in \mathcal{H}\left(\mathbb{O}^{3}\right)$. The group $F_{4}$ is transitive on $\mathbb{O} P^{2}$, and the stabilizer of a point is isomorphic to the spinor group $\operatorname{Spin}(9)$, see [20, Lemma 14.96 and Theorem 14.99]. Hence, $\mathbb{O} P^{2}=F_{4} / \operatorname{Spin}(9)$ is a homogeneous space, and one can prove that $\mathbb{O} P^{2}$ is a two-point homogeneous space.

Now we wish to describe the structure of geodesics in projective spaces. Such a description can be easily given in terms of the models (2.13) and (2.14). It is known, see $\left[8,21,30\right.$, that all geodesics on a two-point homogeneous space $Q\left(d, d_{0}\right)$ are closed and homeomorphic to the unit circle. The group of isometries is transitive on the set of geodesics and the stabilizer of a point is transitive on the set of geodesics passing through this point. Therefore, all geodesics have the same length $2 \pi$ (under the normalization (1.6) ).

The inclusions $\mathbb{R} \subset \mathbb{C} \subset \mathbb{H} \subset \mathbb{O}$ induce the following inclusions of the corresponding projective spaces

$$
\mathbb{F}_{1} P^{n_{1}} \subseteq \mathbb{F} P^{n}, \quad \mathbb{F}_{1} \subseteq \mathbb{F}, \quad n_{1} \leqslant n .
$$

Furthermore, the subspace $\mathbb{F}_{1} P^{n_{1}}$ is a geodesic submanifold in $\mathbb{F} P^{n}$, see [8, Sec. 3.24]. Particularly, the real projective line $\mathbb{R} P^{1}$ is homeomorphic to the unit circle $S^{1}$ and 
can be embedded as a geodesic into all projective spaces $\mathbb{F} P^{n}$,

$$
S^{1} \approx \mathbb{R} P^{1} \subset \mathbb{F} P^{n}
$$

see [8, Proposition 3.32]. In (2.16) $n=2$ if $\mathbb{F}=\mathbb{O}$. These facts can also be immediately derived from a general description of geodesic submanifolds in Riemannian symmetric spaces, see [21, Chap. VII, Corollary 10.5].

Using the models (2.13) and (2.14), we can write the real projective line $\mathbb{R} P^{1}$ as the following set of $2 \times 2$ matrices:

$$
\mathbb{R} P^{1}=\{\zeta(u), u \in \mathbb{R} / \pi \mathbb{Z}\},
$$

where

$$
\zeta(u)=\left(\begin{array}{cc}
\cos ^{2} u & \sin u \cos u \\
\sin u \cos u & \sin ^{2} u
\end{array}\right)=\left(\begin{array}{cc}
\cos u & -\sin u \\
\sin u & \cos u
\end{array}\right)\left(\begin{array}{ll}
1 & 0 \\
0 & 0
\end{array}\right)\left(\begin{array}{cc}
\cos u & \sin u \\
\sin u & \cos u
\end{array}\right) .
$$

For each $u \in \mathbb{R}$ the matrix $\zeta(u)$ is an orthogonal projector onto the one-dimensional subspace $x \mathbb{R}, x=(\cos u, \sin u) \in S^{1}$. The embedding $\mathbb{R} P^{1}$ into $\mathbb{F} P^{n}$ can be written as the following set of $(n+1) \times(n+1)$ matrices

$$
Z=\{Z(u), u \in \mathbb{R} / \pi \mathbb{Z}\} \subset \mathbb{F} P^{n},
$$

where

$$
Z(u)=\left(\begin{array}{cc}
\zeta(u) & 0_{n-1,2} \\
0_{2, n-1} & 0_{n-1, n-1}
\end{array}\right),
$$

and $0_{k, l}$ denotes the zero matrix of size $k \times l$. The set of matrices (2.18) is a geodesic in $\mathbb{F} P^{n}$. All other geodesics are of the form $g(Z)$, where $g \in G$ is an isometry of the space $\mathbb{F} P^{n}$. The parameter $u$ in (2.18) and the geodesic distance $\theta$ on the space $\mathbb{F} P^{n}$ are related by

$$
\theta(Z(u), Z(0))=2|u|, \quad-\pi / 2<u \leqslant \pi / 2,
$$

and for all $u \in \mathbb{R}$ this formula can be extended by periodicity. In particular, we have

$$
\theta(Z(v), Z(-v))=4 v, \quad 0 \leqslant v \leqslant \pi / 4 .
$$

The relation (2.20) will be needed in the next section.

Now, we define the chordal distance on projective spaces. The formulas (2.13), (2.14) and (2.11) imply

$$
\|\Pi\|^{2}=\operatorname{Tr} \Pi^{2}=\operatorname{Tr} \Pi=1 .
$$

for any $\Pi \in \mathbb{F} P^{n}$. Therefore, the projective spaces $\mathbb{F} P^{n}$, defined by (2.13) and (2.14), are submanifolds in the unit sphere

$$
\mathbb{F} P^{n} \subset S^{m-1}=\left\{A \in \mathcal{H}\left(\mathbb{F}^{n+1}\right):\|A\|=1\right\} \subset \mathcal{H}\left(\mathbb{F}^{n+1}\right) \approx \mathbb{R}^{m} .
$$

In fact, the formula (2.22) defines an embedding of $\mathbb{F} P^{n}$ into the $(m-2)$-dimensional sphere, the intersection of the sphere $S^{m-1}$ and the hyperplane in $\mathcal{H}\left(\mathbb{F}^{n+1}\right)$ given by $\operatorname{Tr} A=1$.

The chordal distance $\tau\left(\Pi_{1}, \Pi_{2}\right)$ between $\Pi_{1}, \Pi_{2} \in \mathbb{F} P^{n}$ is defined as the Euclidean distance (2.12):

$$
\tau\left(\Pi_{1}, \Pi_{2}\right)=\frac{1}{\sqrt{2}}\left\|\Pi_{1}-\Pi_{2}\right\|=\left(1-\left\langle\Pi_{1}, \Pi_{2}\right\rangle\right)^{1 / 2} .
$$

The coefficient $1 / \sqrt{2}$ is chosen to satisfy $\operatorname{diam}\left(\mathbb{F} P^{n}, \tau\right)=1$.

It follows from (2.23) that $\tau\left(g\left(\Pi_{1}\right), g\left(\Pi_{2}\right)\right)=\tau\left(\Pi_{1}, \Pi_{2}\right)$ for all isometries $g \in G$ of the space $\mathbb{F} P^{n}$. Since $\mathbb{F} P^{n}$ is a two-point homogeneous space, for any $\Pi_{1}, \Pi_{2} \in$ 
$\mathbb{F} P^{n}$ with $\theta\left(\Pi_{1}, \Pi_{2}\right)=2 u, 0 \leqslant u \leqslant \pi / 2$, there exists an isometry $g \in G$, such that $g\left(\Pi_{1}\right)=Z(u), g\left(\Pi_{2}\right)=Z(0)$. From (2.23), (2.18) and (2.17), we obtain $\tau(Z(u), Z(0))=\sin u=\sin \frac{1}{2} \theta(\Pi(u), \Pi(0))$. Therefore, $\tau\left(\Pi_{1}, \Pi_{2}\right)=\sin \frac{1}{2} \theta\left(\Pi_{1}, \Pi_{2}\right)$, as it was defined before in (1.25). Notice also that pairs of antipodal points $\Pi_{+}, \Pi_{-} \in \mathbb{F} P^{n}$ (with $\theta\left(\Pi_{+}, \Pi_{-}\right)=\pi$ and $\tau\left(\Pi_{+}, \Pi_{-}\right)=1$ ) can be characterized by the orthogonality condition $\left\langle\Pi_{+}, \Pi_{-}\right\rangle=0$, see (2.23).

\section{Proof of Theorem 1.1}

The proof of Theorem 1.1 relies on the following special representation of the symmetric difference metric (1.15).

Lemma 3.1. For a distance-invariant space $\mathcal{M}$, we have

$$
\theta^{\Delta}\left(\xi, y_{1}, y_{2}\right)=\frac{1}{2} \int_{\mathcal{M}}\left|\sigma\left(\theta\left(y_{1}, y\right)\right)-\sigma\left(\theta\left(y_{2}, y\right)\right)\right| \mathrm{d} \mu(y)
$$

with the non-increasing function $\sigma(r)=\xi([r, \pi])=\int_{r}^{\pi} \mathrm{d} \xi(u)$.

In particular, for a homogeneous space $Q=Q\left(d, d_{0}\right)$ and the measure $\mathrm{d} \xi^{\natural}(r)=$ $\sin r \mathrm{~d} r, r \in[0, \pi]$, we have

$$
\theta^{\Delta}\left(\xi^{\natural}, y_{1}, y_{2}\right)=\int_{Q}\left|\tau\left(y_{1}, y\right)^{2}-\tau\left(y_{2}, y\right)^{2}\right| \mathrm{d} \mu(y),
$$

where $\tau$ is the chordal metric (1.25) on $Q\left(d, d_{0}\right)$.

Lemma 3.1 was proved earlier in [24, Lemma 2.1]. Here this result is given in a form adapted to the chordal metric. For the spheres $S^{d}$, a formula similar to (3.2) was given earlier in [10, Lemma 2.4].

Proof of Lemma 3.1. For brevity, we write $\theta\left(y_{1}, y\right)=\theta_{1}$ and $\theta\left(y_{2}, y\right)=\theta_{2}$. Using (1.15), (1.11) and (1.18), we obtain

$$
\begin{aligned}
& \theta^{\Delta}\left(\xi, y_{1}, y_{2}\right) \\
& =\frac{1}{2} \int_{\mathcal{M}}\left(\int_{0}^{\pi}\left(\chi_{0}\left(r-\theta_{1}\right)+\chi_{0}\left(r-\theta_{2}\right)-2 \chi_{0}\left(r-\theta_{1}\right) \chi_{0}\left(r-\theta_{2}\right)\right) \mathrm{d} \xi(r)\right) \mathrm{d} \mu(y) \\
& =\frac{1}{2} \int_{\mathcal{M}}\left(\sigma\left(\theta_{1}\right)+\sigma\left(\theta_{2}\right)-2 \sigma\left(\max \left\{\theta_{1}, \theta_{2}\right\}\right)\right) \mathrm{d} \mu(y) .
\end{aligned}
$$

Since $\sigma$ is a non-increasing function, we have

$$
2 \sigma\left(\max \left\{\theta_{1}, \theta_{2}\right\}\right)=2 \min \left\{\sigma\left(\theta_{1}\right), \sigma\left(\theta_{2}\right)\right\}=\sigma\left(\theta_{1}\right)+\sigma\left(\theta_{2}\right)-\left|\sigma\left(\theta_{1}\right)-\sigma\left(\theta_{2}\right)\right| .
$$

Substituting (3.4) into (3.3), we obtain (3.1).

If $\mathrm{d} \xi^{\natural}(r)=\sin r \mathrm{~d} r$, then the corresponding $\sigma(r)=2-2(\sin r / 2)^{2}$. Substituting this expression into (3.1) and using the definition (1.25), we obtain (3.2).

Proof of Theorem 1.1 for spheres. By (1.26), we have

$$
\begin{gathered}
\tau\left(y_{1}, y\right)^{2}-\tau\left(y_{2}, y\right)^{2}=\frac{1}{4}\left(\left\|y_{1}-y\right\|^{2}-\left\|y_{2}-y\right\|^{2}\right) \\
=\frac{1}{2}\left(y_{2}-y_{1}, y\right)=\tau\left(y_{1}, y_{2}\right)(x, y), \quad y_{1}, y_{2} \in S^{d}
\end{gathered}
$$

where $x=\left\|y_{2}-y_{1}\right\|^{-1}\left(y_{2}-y_{1}\right) \in S^{d}$. Substituting (3.5) into (3.2), we obtain

$$
\theta^{\Delta}\left(\xi^{\natural}, y_{1}, y_{2}\right)=\tau\left(y_{1}, y_{2}\right) \int_{S^{d}}|(x, y)| \mathrm{d} \mu(y) .
$$


The integral in (3.6) is independent of $x \in S^{d}$. This proves the equality (1.29) for $S^{d}$ with the constant

$$
\gamma\left(S^{d}\right)=\left(\int_{S^{d}}|(x, y)| \mathrm{d} \mu(y)\right)^{-1} .
$$

This completes the proof.

The integral (3.7) can be easily calculated to obtain (1.5), see [10,13].

Proof of Theorem 1.1 for real projective spaces. The space $\mathbb{R} P^{d}=S^{d} / S^{0}, S^{0}=$ $\{1,-1\}$ consists of the one dimensional subspaces $y=\mathbf{a} \mathbb{R} \subset \mathbb{R}^{d+1}$, where $\mathbf{a} \in S^{d}$, and $\mathbf{a}$ and $-\mathbf{a}$ are identified. Consider two subspaces $y_{1}=\mathbf{a}_{1} \mathbb{R}, y_{2}=\mathbf{a}_{2} \mathbb{R} \subset \mathbb{R} P^{d}$, and let $\left(\mathbf{a}_{1}, \mathbf{a}_{2}\right) \geqslant 0$. The definitions (1.25) and (2.6) imply

$$
\tau\left(y_{1}, y_{2}\right)^{2}=\left(\sin \frac{1}{2} \theta\left(y_{1}, y_{2}\right)\right)^{2}=1-\left(\mathbf{a}_{1}, \mathbf{a}_{2}\right)^{2} .
$$

Putting $y=\mathbf{a} \mathbb{R}$, we obtain

$$
\begin{aligned}
& \tau\left(y_{2}, y\right)^{2}-\tau\left(y_{1}, y\right)^{2}=\left(\mathbf{a}_{1}, \mathbf{a}\right)^{2}-\left(\mathbf{a}_{2}, \mathbf{a}\right)^{2} \\
& =\left[\left(\mathbf{a}_{1}, \mathbf{a}\right)+\left(\mathbf{a}_{2}, \mathbf{a}\right)\right]\left[\left(\mathbf{a}_{1}, \mathbf{a}\right)-\left(\mathbf{a}_{2}, \mathbf{a}\right)\right]=\left(\mathbf{a}_{1}+\mathbf{a}_{2}, \mathbf{a}\right)\left(\mathbf{a}_{1}-\mathbf{a}_{2}, \mathbf{a}\right) \\
& =2 \sin \frac{1}{2} \theta\left(y_{1}, y_{2}\right)\left(\mathbf{a}_{+}, \mathbf{a}\right)\left(\mathbf{a}_{-}, \mathbf{a}\right)=2 \tau\left(y_{1}, y_{2}\right)\left(\mathbf{a}_{+}, \mathbf{a}\right)\left(\mathbf{a}_{-}, \mathbf{a}\right),
\end{aligned}
$$

where $\mathbf{a}_{1}+\mathbf{a}_{2}=2 \cos \frac{1}{4} \theta\left(y_{1}, y_{2}\right) \mathbf{a}_{+}$and $\mathbf{a}_{1}-\mathbf{a}_{2}=2 \sin \frac{1}{4} \theta\left(y_{1}, y_{2}\right) \mathbf{a}_{-}$. Here $\mathbf{a}_{+}, \mathbf{a}_{-} \epsilon$ $S^{d}$ and $\left(\mathbf{a}_{+}, \mathbf{a}_{-}\right)=0$. The corresponding mutually orthogonal subspaces $y_{+}=$ $\mathbf{a}_{+} \mathbb{R}, y_{-}=\mathbf{a}_{-} \mathbb{R}$ are antipodal points in $\mathbb{R} P^{d}: \theta\left(y_{+}, y_{-}\right)=\pi$.

Substituting (3.8) into (3.2), we obtain

$$
\theta^{\Delta}\left(\xi^{\natural}, y_{1}, y_{2}\right)=2 \tau\left(y_{1}, y_{2}\right) \int_{S^{d} / S^{0}}\left|\left(\mathbf{a}_{+}, \mathbf{a}\right)\left(\mathbf{a}_{-}, \mathbf{a}\right)\right| \mathrm{d} \mu(\mathbf{a}) .
$$

Since $\mathbb{R} P^{d}$ is a two-point homogeneous space, the integral in (3.9) is independent of the choice of mutually orthogonal unit vectors $\mathbf{a}_{+}, \mathbf{a}_{-} \in S^{d}$. This proves the equality (1.29) for $\mathbb{R} P^{d}$ with the constant

$$
\begin{aligned}
\gamma\left(\mathbb{R} P^{d}\right) & =\left(2 \int_{S^{d} / S^{0}}\left|\left(\mathbf{a}_{+}, \mathbf{a}\right)\left(\mathbf{a}_{-}, \mathbf{a}\right)\right| \mathrm{d} \mu(\mathbf{a})\right)^{-1} \\
& =\left(2 \omega_{d}^{-1} \int_{S^{d}}\left|\left(\mathbf{a}_{+}, \mathbf{a}\right)\left(\mathbf{a}_{-}, \mathbf{a}\right)\right| \mathrm{d} \mathbf{a}\right)^{-1},
\end{aligned}
$$

where da denotes the standard surface measure on $S^{d}$ and $\omega_{d}$ is the full surface measure of $S^{d}$. This completes the proof.

The integral in (3.10) can be easily calculated to obtain (1.34) for $\mathbb{R} P^{d}$.

Proof of Theorem 1.1 for general projective spaces. We write $\Pi_{1}, \Pi_{2}, \Pi$ for points in the models of projective spaces (2.13) and (2.14). With this notation, the relation (3.2) takes the form

$$
\theta^{\Delta}\left(\xi^{\natural}, \Pi_{1}, \Pi_{2}\right)=\int_{\mathbb{F} P^{n}}\left|\tau\left(\Pi_{1}, \Pi\right)^{2}-\tau\left(\Pi_{2}, \Pi\right)^{2}\right| \mathrm{d} \mu(\Pi) .
$$


Since $\mathbb{F} P^{n}$ is a two-point homogeneous space, for any $\Pi_{1}, \Pi_{2} \in \mathbb{F} P^{n}$ with $\theta\left(\Pi_{1}, \Pi_{2}\right)=4 v, 0 \leqslant v \leqslant \pi / 4$, there exists an isometry $g \in G$, such that $g\left(\Pi_{1}\right)=$ $Z(v), g\left(\Pi_{2}\right)=Z(-v)$, see (2.20). Therefore,

$$
\int_{\mathbb{F} P^{n}}\left|\tau\left(\Pi_{1}, \Pi\right)^{2}-\tau\left(\Pi_{2}, \Pi\right)^{2}\right| \mathrm{d} \mu(\Pi)=\int_{\mathbb{F} P^{n}}\left|\tau(Z(v), \Pi)^{2}-\tau(Z(-v), \Pi)^{2}\right| \mathrm{d} \mu(\Pi) .
$$

From the definition (2.23), we obtain

$$
\begin{aligned}
\tau(Z(v), \Pi)^{2}-\tau(Z(-v), \Pi)^{2} & =\frac{1}{2}\left(\|Z(v)-\Pi\|^{2}-\|Z(-v)-\Pi\|^{2}\right) \\
& =\langle Z(v)-Z(-v), \Pi\rangle .
\end{aligned}
$$

The formulas (2.17) and (2.18) imply

$$
Z(v)-Z(-v)=\left(\begin{array}{cc}
\zeta(v)-\zeta(-v) & 0_{n-1,2} \\
0_{2, n-1} & 0_{n-1, n-1}
\end{array}\right)
$$

and

$$
\zeta(v)-\zeta(-v)=\left(\begin{array}{cc}
0 & \sin 2 v \\
\sin 2 v & 0
\end{array}\right)=(\sin 2 v)\left(\zeta_{+}-\zeta_{-}\right)
$$

where

$$
\zeta_{+}=\frac{1}{2}\left(\begin{array}{ll}
1 & 1 \\
1 & 1
\end{array}\right), \quad \zeta_{-}=\frac{1}{2}\left(\begin{array}{cc}
1 & -1 \\
-1 & 1
\end{array}\right)
$$

Therefore,

$$
Z(v)-Z(-v)=(\sin 2 v)\left(Z_{+}-Z_{-}\right)
$$

where

$$
Z_{ \pm}=\left(\begin{array}{cc}
\zeta_{ \pm} & 0_{n-1,2} \\
0_{2, n-1} & 0_{n-1, n-1}
\end{array}\right)
$$

We have $Z_{ \pm}^{*}=Z_{ \pm}, Z_{ \pm}^{2}=Z_{ \pm}$, $\operatorname{Tr} Z_{ \pm}=1$, Therefore, $Z_{ \pm} \in \mathbb{F} P^{n}$, and $\left\langle Z_{+}, Z_{-}\right\rangle=0$. This means that $Z_{+}$and $Z_{-}$are antipodal points in $\mathbb{F} P^{n}$. Using (1.25), we can write

$$
\tau\left(\Pi_{1}, \Pi_{2}\right)=\tau(Z(v), Z(-v))=\sin 2 v,
$$

and the equality (3.14) takes the form

$$
Z(v)-Z(-v)=\tau\left(\Pi_{1}, \Pi_{2}\right)\left(Z_{+}-Z_{-}\right) .
$$

Substituting (3.15) into (3.13), we find that

$$
\tau(Z(v), \Pi)^{2}-\tau(Z(-v), \Pi)^{2}=\tau\left(\Pi_{1}, \Pi_{2}\right)\left\langle Z_{+}-Z_{-}, \Pi\right\rangle .
$$

Substituting (3.16) into (3.12) and using (3.11), we obtain

$$
\theta^{\Delta}\left(\xi^{\natural}, \Pi_{1}, \Pi_{2}\right)=\tau\left(\Pi_{1}, \Pi_{2}\right) \theta^{\Delta}\left(\xi^{\natural}, Z_{+}, Z_{-}\right),
$$

where

$$
\theta^{\Delta}\left(\xi^{\natural}, Z_{+}, Z_{-}\right)=\int_{\mathbb{F} P^{n}}\left|\left\langle Z_{+}-Z_{-}, \Pi\right\rangle\right| \mathrm{d} \mu(\Pi) .
$$

The integral (3.18) is independent of $\Pi_{1}$ and $\Pi_{2}$. This proves the equality (1.29) for $\mathbb{F} P^{n}$ with the constant

$$
\gamma\left(\mathbb{F} P^{n}\right)=\left(\int_{\mathbb{F} P^{n}}\left|\left\langle Z_{+}-Z_{-}, \Pi\right\rangle\right| \mathrm{d} \mu(\Pi)\right)^{-1} .
$$

Notice that in this formula any pair of antipodal points in $\mathbb{F} P^{n}$ can be taken instead of $Z_{+}, Z_{-}$. The proof of Theorem 1.1 is complete. 


\section{Proof of Theorem 1.2}

The zonal spherical functions $\phi_{l}$ for the spaces $Q=Q\left(d, d_{0}\right)$ are eigenfunctions of the radial part of the Laplace-Beltrami operator on $Q$ and can be given explicitly, see [18, p. 178], 22, Chap. V, Theorem 4.5], 23, pp. 514-512, 543-544], [31, Theorem 11.4.21]. We have

$$
\phi_{l}\left(x_{1}, x_{2}\right)=\phi_{l}\left(Q, x_{1}, x_{2}\right)=\frac{P_{l}^{(\alpha, \beta)}\left(\cos \theta\left(x_{1}, x_{2}\right)\right)}{P_{l}^{(\alpha, \beta)}(1)}, \quad l \geqslant 0, x_{1}, x_{2} \in Q,
$$

where $P_{l}^{(\alpha, \beta)}(t), t \in[-1,1]$, are Jacobi polynomials of degree $l$ with parameters

$$
\alpha=d / 2-1, \quad \beta=d_{0} / 2-1, \quad \alpha, \beta \geqslant-1 / 2 .
$$

A detailed consideration of Jacobi polynomials can be found in [3,4,28. They can be given by Rodrigues' formula

$$
P_{l}^{(\alpha, \beta)}(t)=\frac{(-1)^{l}}{2^{l} l !}(1-t)^{-\alpha}(1+t)^{-\beta} \frac{d^{l}}{d t^{l}}\left\{(1-t)^{l+\alpha}(1+t)^{l+\beta}\right\} .
$$

We also have the bound

$$
\left|P_{l}^{(\alpha, \beta)}(t)\right| \leqslant P_{l}^{(\alpha, \beta)}(1)=\left(\begin{array}{c}
\alpha+l \\
l
\end{array}\right)=O\left(l^{\alpha}\right), \quad t \in[-1,1],
$$

see [28, Theorem 7.32.1].

Jacobi polynomials form a complete orthogonal system in the $L_{2}$-space on the segment $[-1,1]$ with the weight $(1-t)^{\alpha}(1+t)^{\beta}$. We have the orthogonality relations,

$$
\int_{-1}^{1} P_{l}^{(\alpha, \beta)}(t) P_{l^{\prime}}^{(\alpha, \beta)}(t)(1-t)^{\alpha}(1+t)^{\beta} \mathrm{d} t=\delta_{l l^{\prime}} 2^{\alpha+\beta+1} M_{l}^{-1},
$$

where $\delta_{l l^{\prime}}$ is Kronecker's symbol and

$$
M_{l}=(2 l+\alpha+\beta+1) \frac{\Gamma(l+1) \Gamma(l+\alpha+\beta+1)}{\Gamma(l+\alpha+1) \Gamma(l+\beta+1)}=O(l),
$$

see [28, Eq. (4.3.3)]. Notice that the asymptotic approximations in (4.4) and (4.6) follow easily from Stirling's formula.

Using the orthogonality relations (4.5), we obtain the following formal expansion

$$
f(t)=\sum_{l \geqslant 0} 2^{-\alpha-\beta-1} M_{l} c_{l} P_{l}^{(\alpha, \beta)}(t),
$$

for an integrable function $f(t), t \in[-1,1]$, where

$$
c_{l}=\int_{-1}^{1} f(t)(1-t)^{\alpha}(1+t)^{\beta} P_{l}^{(\alpha, \beta)}(t) \mathrm{d} t
$$

If all derivatives $f^{(l)}(t)$ exist, and $f^{(l)}(t)(1-t)^{\alpha+l}(1+t)^{\beta+l}$ vanish at $t= \pm 1$ for all $l$, then substituting Rodrigues' formula (4.3) into (4.8) and integrating $l$ times by part, we obtain

$$
c_{l}=\frac{1}{2^{l} l !} \int_{-1}^{1} f^{(l)}(t)(1-t)^{\alpha+l}(1+t)^{\beta+l} \mathrm{~d} t .
$$

In what follows, we always assume that the parameters $\alpha, \beta$ and the dimensions $d, d_{0}$ are related by (4.2). We shall also use the well-known formulas for the beta 
function

$$
\begin{aligned}
B(a, b)=\frac{\Gamma(a) \Gamma(b)}{\Gamma(a+b)} & =\int_{0}^{\pi}\left(\sin \frac{1}{2} u\right)^{2 a-1}\left(\cos \frac{1}{2} u\right)^{2 b-1} \mathrm{~d} u \\
& =2^{1-a-b} \int_{-1}^{1}(1-t)^{a-1}(1+t)^{b-1} \mathrm{~d} t
\end{aligned}
$$

and the following notation

$$
(a)_{0}=1,(a)_{l}=a(a+1) \ldots(a+l-1)=\frac{\Gamma(\alpha+l)}{\Gamma(\alpha)}
$$

for the falling factorial, see [4, Sec.10.7, Eq.(11)].

Lemma 4.1. For any space $Q=Q\left(d, d_{0}\right)$, the chordal metric $\tau$ has the following zonal spherical function expansion

$$
\tau\left(y_{1}, y_{2}\right)=\frac{1}{2} \sum_{l \geqslant 1} M_{l} C_{l}\left[1-\phi_{l}\left(Q, x_{1}, x_{2}\right)\right]
$$

where

$$
C_{l}=B(\alpha+3 / 2, \beta+l+1) \Gamma(l+1)^{-1}(1 / 2)_{l-1} P_{l}^{(\alpha, \beta)}(1) .
$$

The series (4.12) converges absolutely and uniformly.

Proof. Applying the formulas (4.7) - (4.9) to the function $f(t)=(1-t)^{1 / 2}$ and using (4.10), we obtain the expansion

$$
\begin{aligned}
(1-t)^{1 / 2} & =2^{1 / 2} \Gamma(\alpha+3 / 2) \times \\
& \sum_{l \geqslant 0} \frac{(2 l+\alpha+\beta+1) \Gamma(l+\alpha+\beta+1)(-1 / 2)_{l}}{\Gamma(l+\alpha+1) \Gamma(l+\alpha+\beta+5 / 2)} P_{l}^{(\alpha, \beta)}(t) .
\end{aligned}
$$

Taking into account (4.4) and (4.11), and applying Stirling's approximation to the gamma functions in (4.14), we observe that the coefficients in (4.14) are of the order $O\left(l^{-2}\right)$. Therefore, the series (4.14) converges absolutely and uniformly.

Since $(-1 / 2)_{0}=1$ and $(-1 / 2)_{l}=-1 / 2(1 / 2)_{l-1}$ for $l \geqslant 1$, the series (4.14) can be written as follows

$$
\left(\frac{1-t}{2}\right)^{1 / 2}=M_{0} C_{0}-\frac{1}{2} \sum_{l \geqslant 1} M_{l} C_{l} \frac{P_{l}^{(\alpha, \beta)}(t)}{P_{l}^{(\alpha, \beta)}(1)},
$$

where $C_{0}=B(\alpha+3 / 2, \beta+1)$ and $C_{l}, l \geqslant 1$ are given in (4.13). Putting $t=1$, we find

$$
M_{0} C_{0}=\frac{1}{2} \sum_{l \geqslant 1} M_{l} C_{l} .
$$

Combining (4.15) and (4.16), we obtain

$$
\left(\frac{1-t}{2}\right)^{1 / 2}=\frac{1}{2} \sum_{l \geqslant 1} M_{l} C_{l}\left[1-\frac{P_{l}^{(\alpha, \beta)}(t)}{P_{l}^{(\alpha, \beta)}(1)}\right] .
$$

For $t=\cos \theta\left(x_{1}, x_{2}\right)$, the equality (4.17) coincides with (4.12).

Remark 4.1. The expansion (4.14) can be found in [4, Sec.10.20, Eq.(3)]. However, it should be noted that the linear co-factor $(2 l+\alpha+\beta+1)$ in (4.14) is misprinted in [4, Sec.10.20, Eq.(3)] as $\Gamma(2 l+\alpha+\beta+1)$. 
Lemma 4.2. For any space $Q=Q\left(d, d_{0}\right)$ and any finite measure $\xi$ on $[0, \pi]$, the symmetric difference metric (1.15) has the following zonal spherical function expansion

$$
\theta^{\Delta}\left(\xi, y_{1}, y_{2}\right)=B\left(d / 2, d_{0} / 2\right)^{-1} \sum_{l \geqslant 1} l^{-2} M_{l} A_{l}(\xi)\left[1-\phi_{l}\left(Q, x_{1}, x_{2}\right)\right],
$$

where

$$
A_{l}(\xi)=\int_{0}^{\pi}\left(\sin \frac{1}{2} r\right)^{2 d}\left(\cos \frac{1}{2} r\right)^{2 d_{0}}\left\{P_{l-1}^{(\alpha+1, \beta+1)}(\cos r)\right\}^{2} \mathrm{~d} \xi(r) .
$$

The series (4.18) converges absolutely and uniformly.

The expansion (4.18) has been established in 25, Theorem 4.1(ii)]. The proof is based on the observation that the term $\mu\left(\mathcal{B}\left(y_{1}, r\right) \cap \mathcal{B}\left(y_{2}, r\right)\right)$ in the formula (1.19) can be thought of as a convolution of the characteristic functions of the balls on the homogeneous space $Q\left(d, d_{0}\right)$.

Proof of Theorem 1.2. Substituting (4.12) and (4.18) into (1.29) and equating coefficients at each $\phi_{l}$, we obtain the following series of equations

$$
\gamma(Q) A_{l}\left(\xi^{\natural}\right)=\frac{(1 / 2)_{l-1} l^{2}}{2 \Gamma(l+1)}\left(\begin{array}{c}
\alpha+l \\
l
\end{array}\right) B\left(d / 2, d_{0} / 2\right) B\left((d+1) / 2, l+d_{0} / 2\right),
$$

for all $l \geqslant 1$. If the measure $\mathrm{d} \xi^{\natural}(r)=\sin (r) \mathrm{d} r$, the integral (4.19) takes the form

$$
A_{l}\left(\xi^{\natural}\right)=2 \int_{0}^{\pi}\left(\sin \frac{1}{2} r\right)^{2 d+1}\left(\cos \frac{1}{2} r\right)^{2 d_{0}+1}\left\{P_{l-1}^{(\alpha+1, \beta+1)}(\cos r)\right\}^{2} \mathrm{~d} r .
$$

Each of the equations (4.20) can be used to determine the constant $\gamma(Q)$. In the simplest case of $l=1$, we have

$$
\gamma(Q) A_{1}\left(\xi^{\natural}\right)=\frac{d}{4} B\left(d / 2, d_{0} / 2\right) B\left((d+1) / 2,1+d_{0} / 2\right),
$$

where

$$
A_{1}\left(\xi^{\natural}\right)=2 \int_{0}^{\pi}\left(\sin \frac{1}{2} r\right)^{2 d+1}\left(\cos \frac{1}{2} r\right)^{2 d_{0}+1} \mathrm{~d} r=2 B\left(d+1, d_{0}+1\right),
$$

see (4.10). Therefore,

$$
\gamma(Q)=\frac{d B\left(d / 2, d_{0} / 2\right) B\left((d+1) / 2,1+d_{0} / 2\right)}{8 B\left(d+1, d_{0}+1\right)} .
$$

In the terms of gamma functions, we have

$$
\gamma(Q)=\frac{\Gamma(d / 2) \Gamma\left(d_{0} / 2\right)^{2} \Gamma((d+1) / 2) \Gamma\left(d+d_{0}+2\right)}{16 \Gamma(d) \Gamma\left(d_{0}\right) \Gamma\left(\left(d+d_{0}\right) / 2\right) \Gamma\left(\left(d+d_{0}+3\right) / 2\right)},
$$

where the relation $\Gamma(z+1)=z \Gamma(z)$ has been used. Applying the duplication formula $\Gamma(2 z)=\pi^{-1 / 2} 2^{2 z-1} \Gamma(z) \Gamma(z+1 / 2)$ to the terms $\Gamma(d), \Gamma\left(d_{0}\right)$ and $\Gamma\left(d+d_{0}+2\right)$, we obtain

$$
\gamma(Q)=\frac{\sqrt{\pi}}{4}\left(d+d_{0}\right) \frac{\Gamma\left(d_{0} / 2\right)}{\Gamma\left(\left(d_{0}+1\right) / 2\right)} .
$$

This completes the proof. 
Remark 4.2. The equalities (4.20) with the constant (4.26) define explicit formulas for the integrals (4.21). We have

$$
\begin{aligned}
\int_{-1}^{1}\left(P_{l-1}^{\left(d / 2, d_{0} / 2\right)}(t)\right)^{2} & \left(\frac{1-t}{2}\right)^{d}\left(\frac{1+t}{2}\right)^{d_{0}} \mathrm{~d} t=\frac{2(1 / 2)_{l-1}}{(l-1) !} B\left(d+1, d_{0}+1\right) \times \\
& \times \frac{\left.\Gamma(d / 2+l) \Gamma\left(d_{0} / 2+l\right) \Gamma\left(d / 2+d_{0} / 2+3 / 2\right)\right)}{\Gamma(d / 2+1) \Gamma\left(d_{0} / 2+1\right) \Gamma\left(d / 2+d_{0} / 2+1+l\right)}
\end{aligned}
$$

for all $l \geqslant 1$.

It is worth noting that a direct proof of the formulas (4.27) makes possible an alternative proof of Theorems 1.1 and 1.2. Indeed, the formulas (4.27) together with the expansions (4.12) and (4.18) imply the relations (1.29) and (1.32). Such an approach to the proof of invariance principle will be realized in the second part of this work.

\section{REFERENCES}

[1] J. R. Alexander, On the sum of distances between $n$ points on a sphere. Acta Math. Hungar., 23 (3-4) (1972), 443-448.

[2] J. R. Alexander, J. Beck, W. W. L. Chen, Geometric discrepancy theory and uniform distributions. - in Handbook of Discrete and Computational Geometry (J. E. Goodman and J. O'Rourke eds.), Chapter 10, pages 185-207, CRC Press LLC, Boca Raton, FL, 1997.

[3] G. E. Andrews, R. Askey, R. Roy, Special functions. Cambridge Univ. Press, 2000.

[4] H. Bateman, A. Erdélyi, Higher transcendental functions, vol. 2, Mc Graw-Hill Book Comp. Inc., New York, Toronto, London, 1953.

[5] J. C. Baez, Octonions, Bull. Amer. Math. Soc., 39, (2002), 145-205; errata in Bull. Amer. Math. Soc., 42 (2005), 213.

[6] J. Beck, Sums of distances between points on a sphere: An application of the theory of irregularities of distributions to distance geometry, Mathematika, 31, (1984), 33-41.

[7] J. Beck, W. W. L. Chen, Irregularities of Distribution. Cambridge Tracts in Math., vol. 89, Cambridge Univ. Press, 1987.

[8] A. L. Besse, Manifolds all of whose geodesics are closed, A series of modern surveys in Math., vol. 93, Springer, 1978.

[9] D. Bilyk, M. Lacey, One bit sensing, discrepancy, and Stolarsky principle, Sbornik Math., 208(6), (2017), 744-763.

[10] D. Bilyk, F. Dai, R. Matzke. Stolarsky principle and energy optimization on the sphere, Constr. Approx., 48(1), (2018), 31-60.

[11] D. Bilyk, F. Dai, Geodesic distance Riesz energy on the sphere, Trans. AMS, 372 (2018), 3141-3166.

[12] L. Brandolini, W.W.L. Chen, L. Colzani, G. Gigante, G. Travaglini. Discrepancy and numerical integration on metric measure spaces. J. Geometric Anal., 29(1), (2019), 328-369.

[13] J. S. Brauchart, J. Dick, A simple proof of Stolarsky's invariance principle. — Proc. Amer. Math. Soc., 141, (2013), 2085-2096.

[14] H. Cohn, A. Kumar, Universally optimal distribution of points on spheres, J. Amer. Math. Soc., 20, (2006), 99-147.

[15] H. Cohn, A. Kumar, G. Minton, Optimal simplices and codes in projective spaces, (2013), Geometry and Topology, 20 (2016), 1289-1357.

[16] J. Conway, R. Hardin, N. J. A. Sloane, Packing lines, planes, etc.: packing in Grassmannian spaces, Experiment. Math., 5, (1996), 139-159.

[17] M. M. Deza, M. Laurent, Geometry of cuts and metrics, Springer, 1997.

[18] R. Gangolli, Positive definite kernels on homogeneous spaces and certain stochastic processes related to Lévy's Brownian motion of several parameters, Ann. Inst. Henri Poincaré, vol. III, No. 2, (1967), 121-325.

[19] A. Gray, The volume of a small geodesic ball of a Riemannian manifold, Michigan Math. J., 20 (1974), 329-344. 
[20] F. R. Harvey, Spinors and calibrations, Academic Press, 1990.

[21] S. Helgason, Differential Geometry, Lie Groups, and Symmetric Spaces, Academic Press, 1978.

[22] S. Helgason, Groups and geometric analysis. Integral geometry, invariant differential operators, and spherical functions, Academic Press, 1984.

[23] V. I. Levenshtein, Universal bounds for codes and designs, in Handbook of Coding Theory (V. S. Pless and W. C. Huffman eds.), Chapter 6, pages 499-648, Elsevier, 1998.

[24] M. M. Skriganov, Point distributions in compact metric spaces, Mathematika, 63, (2017), $1152-1171$.

[25] M. M. Skriganov, Point distributions in two-point homogeneous spaces, Mathematika, 65, (2019), 557-587.

[26] M. M. Skriganov, Bounds for $L_{p}$-discrepancies of point distributions in compact metric measure spaces, Constr. Approx., (2019), published on line, https://doi.org/10.1007/s00365-01909476-z.

[27] K. B. Stolarsky, Sums of distances between points on a sphere, II, Proc. Amer. Math. Soc., 41, (1973), 575-582.

[28] G. Szegö , Orthogonal polynomials, Amer. Math. Soc., 1950.

[29] N. Ja. Vilenkin, A. U. Klimyk, Representation of Lie groups and special functions, vols. 1-3, Kluwer Acad. Pub., Dordrecht, 1991-1992.

[30] J. A. Wolf, Spaces of constant curvature, Univ. Califormia, Berkley, 1972.

[31] J. A. Wolf, Harmonic analysis on commutative spaces, Math. Surveys and Monographs, vol. 142, Amer. Math. Soc., 2007.

St. Petersburg Department of the Steklov Mathematical Institute of the Russian Academy of Sciences, 27, Fontanka, St.Petersburg 191023, Russia

E-mail address: mmskrig@gmail.com 